DR. ALBERTO AIMO (Orcid ID : 0000-0001-9129-9519)

PROF. RAFFAELE DE CATERINA (Orcid ID : 0000-0003-1637-574X)

Article type : Original Paper

\title{
Pre-Treatment High-Sensitivity Troponin T for the Short-Term Prediction of Cardiac Outcomes in Patients on Immune Checkpoint Inhibitors
}

Short title: Hs-troponin T and cardiac outcomes in patients on ICl
Serena Petricciuoloํ․, Maria Grazia Delle Donne ${ }^{1}$, Alberto Aimo ${ }^{1,2}$, Antonio Chella ${ }^{3}$, and Raffaele De Caterina ${ }^{1}$

1. Cardiology Division, Pisa University Hospital, Pisa, Italy; 2. Institute of Life Sciences, Scuola Superiore Sant'Anna, Pisa, Italy; 3. Pneumo-Oncology Unit, Pisa University Hospital, Pisa, Italy

Word count: 2587 (text)

Conflicts of interest: none

Address for correspondence:

Prof. Raffaele De Caterina, MD, PhD

Chair of Cardiology, University of Pisa, and Cardiovascular Division, Pisa University Hospital

Via Paradisa 2

56124 Pisa, Italy

Tel.: +39-050-996-751

E-mail: raffaele.decaterina@unipi.it

This article has been accepted for publication and undergone full peer review but has not been through the copyediting, typesetting, pagination and proofreading process, which may lead to differences between this version and the Version of Record. Please cite this article as doi: $\underline{10.1111 / \mathrm{ECI} .13400}$

This article is protected by copyright. All rights reserved 


\section{ABSTRACT}

Background: Immune checkpoint inhibitors (ICls) are an emerging option for several advanced metastatic cancers, but may have cardiotoxic effects. The prognostic value of high-sensitivity troponin T (hs-TnT) before treatment start has never been investigated.

Materials and Methods: Thirty consecutive patients underwent measurement of hs-TnT before starting $\mathrm{ICl}$ therapy (pembrolizumab, 23\%; nivolumab, 12\%; atezolizumab, 6\%; durvalumab, 5\%). The primary (cardiovascular death, stroke or transient ischemic attack, pulmonary embolism and new-onset heart failure) and secondary endpoints (progression of cardiac involvement according to the CARDIOTOX classification) were evaluated after 3 months from the first cycle.

Results: Patients (median age 68 years, 77\% men, 13\% with coronary artery disease, 90\% current or former smokers, $67 \%$ overweight or obese, and $43 \%$ hypertensive) had a median hs$\mathrm{TnT}$ of $12 \mathrm{ng} / \mathrm{L}$ (interquartile interval 8-23). The primary endpoint occurred only in patients with hs-TnT $\geq 14 \mathrm{ng} / \mathrm{L}$ at baseline. Therefore, only patients who had hs-TnT $\geq 14 \mathrm{ng} / \mathrm{L}$ before the first cycle died, had a stroke/TIA, or new-onset HF. Furthermore, 9 out of 13 patients with the secondary endpoint (progression of cardiac disease) had hs-TnT $\geq 14 \mathrm{ng} / \mathrm{L}$ before the first cycle $(p=0.012)$. AUC values were 0.909 for the primary endpoint, and 0.757 for the secondary endpoint. The best cut-off was $14 \mathrm{ng} / \mathrm{L}$ for both the primary (100\% sensitivity, $73 \%$ specificity) and secondary endpoints (sensitivity $75 \%$, specificity $77 \%$ ).

Conclusions: In patients on ICls, baseline hs-TnT predicts a composite cardiovascular endpoint and the progression of cardiac involvement at 3 months, with $14 \mathrm{ng} / \mathrm{L}$ as the best cut-off.

Word count: 245 (abstract).

Keywords: immune checkpoint inhibitors; cardiotoxicity; cardiac troponin; prediction. 


\section{INTRODUCTION}

The development of immunotherapies has revolutionized the management of an increasing number of advanced-stage malignancies with poor prognosis ${ }^{1-3}$. Seven immune checkpoint inhibitors (ICls) have been approved by the Food and Drug Administration: ipilimumab (an inhibitor of cytotoxic T-lymphocyte-associated antigen 4 [CTLA-4]), nivolumab, cemipilimab and pembrolizumab (inhibitors of programmed cell death protein 1 [PD-1]); atezolizumab, avelumab, and durvalumab (inhibitors of programmed cell death ligand 1 [PD-L1]) ${ }^{4}$. These drugs have shown remarkable results in treating advanced metastatic cancers, including non-small cell lung cancer, renal cell carcinoma, melanoma and squamous cell carcinoma, and refractory Hodgkin's lymphoma ${ }^{5}$.

By removing important brakes to the inflammatory response, therapy with $\mathrm{ICI}$ may trigger autoimmune manifestations, possibly involving also the heart ${ }^{6,7}$. The first case of fulminant myocarditis shortly after ICI treatment was reported in $2016^{\circ}$. In a recent registry study, patients on $\mathrm{ICl}$ had an 11-fold higher likelihood of myocarditis ${ }^{9}$, and a cohort study of 964 patient from a multicentre registry reported a prevalence of $1.14 \%$, which increased to as high as $2.4 \%$ for combination therapy with anti-PD-1/anti-CTLA-4 ${ }^{10}$. ICl-associated myocarditis then appears to be a class effect, and its incidence seems to be higher when patients receive a combination therapy ${ }^{8,10}$. Other inflammatory cardiovascular adverse events have been also associated with $\mathrm{ICI}$ treatment, particularly pericardial disease and vasculitis ${ }^{9}$. Non-inflammatory cardiac adverse events (AEs) have also been reported, although their incidence and mechanisms are still poorly defined. These include myocardial infarction ${ }^{11}$, coronary vasospasm ${ }^{12}$, asymptomatic noninflammatory left ventricular (LV) dysfunction ${ }^{13}$, a Takotsubo-like syndrome ${ }^{14-16}$, and arrhythmias or conduction disturbances ${ }^{17,18}$.

High-sensitivity troponins are sensitive and specific indicators of cardiomyocyte damage ${ }^{19}$, and strong predictors of outcome in different settings, including chronic heart failure $(\mathrm{HF})^{20}$, subjects in the general population ${ }^{21}$, and also patients receiving cardiotoxic chemotherapies such as anthracyclines ${ }^{22,23}$. Mahmood et al. showed how useful for surveillance is the measurement of hs$\mathrm{TnT}$ levels at baseline and after each cycle of ICI treatment, with this parameter being abnormal in $94 \%$ of ICl-myocarditis patients at clinical presentation ${ }^{10}$. Conversely, Sarocchi et al. measured

TnT levels at each nivolumab administration in 59 patients, and found elevations in only 6 patients, none of whom developed overt cardiac adverse events ${ }^{24}$. These researchers mentioned possible reasons for "false positive" hs-TnT elevations, including a myocardial oxygen demandsupply mismatch due to worsening of the clinical status or subclinical ICl-induced myocarditis ${ }^{24}$.

This article is protected by copyright. All rights reserved 
While the role of hs-TnT for early detection of cardiac involvement remains to be clarified, it is interesting to notice that baseline elevation of hs-TnT (i.e., before the first cycle of $\mathrm{ICI}$ ), reflecting a condition of myocardial vulnerability and then a greater risk of cardiotoxicity, has never been evaluated as a possible predictor of outcome, as previously demonstrated for anthracyclines ${ }^{25}$ or trastuzumab ${ }^{26}$. In this study we assessed this hypothesis for the first time.

\section{MATERIALS AND METHODS}

\section{Patient population}

Thirty consecutive patients starting a treatment with ICl from September 2019 to March 2020 were prospectively enrolled in a dedicated outpatient clinic of the University Hospital of Pisa. The following inclusion criteria were considered: age $\geq 18$ years; histological or cytological diagnosis of squamous cell lung cancer, adenocarcinoma, neuroendocrine lung cancer, or pleural mesothelioma; tumour stage IIIb or IV. Patients had been previously screened for asymptomatic left ventricular (LV) systolic dysfunction (LV ejection fraction [LVEF] $<50 \%$ ) or clinical HF, which represented exclusion criteria.

Following baseline evaluation (see below), patients were started on ICl. The specific drug was chosen by the treating oncologist according to current indications ${ }^{27}$. Pembrolizumab was administered intravenously (iv) at the dose of $200 \mathrm{mg}$ iv every 21 days, nivolumab at $240 \mathrm{mg}$ iv every 15 days, durvalumab at $10 \mathrm{mg} / \mathrm{kg}$ iv every 44 days, and atezolizumab $1200 \mathrm{mg}$ iv every 21 days.

\section{Baseline characterization: venous blood sampling, ECG, echocardiogram}

Within 24 hours before the start of ICI therapy, patients underwent venous blood sampling, a standard 12-lead electrocardiogram (ECG), and a transthoracic echocardiogram (TTE). The results of these investigations did not influence the treatment plan.

Blood samples were drawn at 8 A.M. after an overnight fasting period and a 20-minute supine rest, as previously described ${ }^{26}$. Estimated glomerular filtration rate (eGFR) was calculated from plasma creatinine based on the Chronic Kidney Disease Epidemiology Collaboration (CKD-EPI) formula ${ }^{29}$. hs-TnT was measured on frozen serum aliquots with a homogeneous sandwich immunoassay based on the ElectroChemiLuminiscence ImmunoAssay (ECLIA) technique on a COBAS apparatus (Roche Diagnostics; limit of blank $3 \mathrm{ng} / \mathrm{L}$, upper reference limit $14 \mathrm{ng} / \mathrm{L}$ ) ${ }^{30}$.

Standard, two-dimensional TTE images were obtained using a Philips IE33 Ultrasound machine, with X5-1 transducer (Philips Medical Systems, Palo Alto, California, USA). Wall thickness, chamber volumes, and indices of systolic and diastolic function were assessed according to the 
American Society of Echocardiography and European Association of Cardiovascular Imaging guidelines, with volumes measured with the biplane method of disks (modified Simpson's rule) ) $^{31-}$ 35. We also used 2D speckle tracking echocardiography (frame rate 45-90 frames/s) to asses LV global longitudinal strain (GLS), according to current standards ${ }^{36}$. Data sets were digitally stored, exported to a workstation equipped with a commercial software (Philips QLAB), and interpreted according to the American Society of Echocardiography/European Association of Cardiovascular Imaging Expert Consensus Document ${ }^{37}$.

\section{Follow-up}

During follow-up, the presence and severity of myocardial injury was classified according to the CARDIOTOX classification:

- normal: no evidence of myocardial injury/dysfunction. Asymptomatic patients with normal biomarkers and LV function parameters;

- mild: asymptomatic patients with left ventricular ejection fraction (LVEF) $\geq 50 \%$ with elevated biomarkers or at least one additional abnormal echo parameter (increased LV end-systolic volume, left atrial (LA) area $>30 \mathrm{~cm}^{2}, 10 \%$ decrease of LVEF to an LVEF $<53 \%$, average E/e' $>14$, GLS $>-18 \%, 15 \%$ relative reduction of GLS from baseline);

- moderate: asymptomatic patients with LVEF $\geq 40 \%$ and $<50 \%$ with or without biomarker increase or other LV function abnormalities;

- severe: patients with asymptomatic LVEF $<40 \%$ or clinical $\mathrm{HF}^{38}$.

As the timing of patient re-evaluation was not specified in the study introducing this classification $^{38}$, we chose to perform a cardiological re-evaluation including clinical history, physical examination, and TTE immediately before each therapy cycle.

ICl-related AEs were searched and managed according to current recommendations ${ }^{39}$. In addition to planned visits, patients were re-evaluated in the ambulatory clinic or hospitalized when clinically indicated. Collection of follow-up data was integrated by phone calls to patients or their relatives. The primary composite endpoint (which included cardiovascular death, non-fatal myocardial infarction [MI], stroke or transient ischemic attack [TIA], pulmonary embolism, and new-onset HF) was evaluated after 3 months from the first cycle. The secondary endpoint was progression of cardiac involvement according to the CARDIOTOX classification (for example, from normal to mild disease $)^{38}$, evaluated again at 3 months.

\section{Statistical analysis}

Statistical analysis was performed using the IBM SPSS Statistics (version 22, 2013) package and the R statistical software (http://www.r-project.org/, version 3.4.4). Normal distribution was 
assessed with the Kolmogorov-Smirnov test; as all continuous variables had a non-normal distribution, they were expressed as medians and interquartile intervals. Differences between groups were evaluated through the Mann-Whitney U-test, while categorical variables were compared by the Chi-square test with the Yates correction. hs-TnT levels were $\log _{2}$-transformed to account for skewed distribution. Area under the curve (AUC) values of $\log _{2}(\mathrm{hs}-\mathrm{TnT})$ for the prediction of the 3-month primary and secondary endpoints were calculated, and best cut-offs were searched through the Youden analysis.

$\log _{2}(\mathrm{hs}-\mathrm{TnT})$ was evaluated as possible predictor of the primary and secondary endpoints by univariable logistic regression analysis. Multivariable analyses for outcome prediction were not performed because of the low number of events, in agreement with the "one-in-ten" rule ${ }^{40}$. Predictors of circulating levels of ( $\log _{2}$-transformed) hs-TnT were then searched among all available baseline variables through linear regression analysis. Univariable predictors with $p$ $<0.10$ were entered into a multivariable model; multicollinearity was excluded by calculating the variance inflation factor. Two-tailed $p$ values $<0.05$ were considered as statistically significant.

\section{RESULTS}

\section{Patient population}

Main characteristics of the study population are reported in Table 1. Patients were aged 68 years (59-75), and were most often men (77\%). A minority of patients (13\%) had known coronary artery disease, but cardiovascular risk factors were fairly well represented, particularly current or former smoking status (90\% overall), overweight or obesity $(67 \%)$, and hypertension $(43 \%)$. Renal function and echocardiographic parameters were within normal limits. Hs-TnT and GLS were both normal (12 ng/L [8-23] and $-17 \%$ [-20 to -15$]$, respectively). The most common diagnosis was adenocarcinoma $(63 \%)$, followed by squamous cell lung cancer $(20 \%)$. The majority of patients were on pembrolizumab (23\%), followed by nivolumab (12\%). As for cardiovascular therapies, $37 \%$ of patients were receiving aspirin, $33 \%$ were on statins, $27 \%$ on a beta-blocker $(27 \%)$ and $33 \%$ on a calcium channel blocker (Table 1).

When stratifying patients based on the upper reference limit of hs-TnT (14 ng/L), patients with hsTnT were older, had more often hypertension, diabetes and peripheral artery disease, and were more often current or previous smokers. They had also a lower eGFR and a higher LA volume index (LAVi), although both remaining within normal limits. Additionally, patients with hs-TnT $\geq 14$ $\mathrm{ng} / \mathrm{L}$ were more often on aspirin (Table 1 ).

\section{Outcome}


At the 3-month timepoint, 7 patients (23\%) had experienced the primary endpoint. Specifically, 2 patients $(7 \%)$ died for cardiovascular causes, one for $\mathrm{Ml}$ and the other for pericardial tamponade; other 2 patients (7\%) had a stroke or TIA, and $3(10 \%)$ developed HF. Only one other patient died for non-cardiovascular causes. As for the secondary endpoint, 13 patients (43\%) displayed a progression of cardiac involvement according to the CARDIOTOX classification over 3 months: 3 $(10 \%)$ from normal to mild, 1 (3\%) from normal to moderate, $5(17 \%)$ from mild to moderate, and $4(13 \%)$ from mild to severe.

\section{Troponin and patient outcome}

The primary endpoint occurred only in patients with $\mathrm{hs}-\mathrm{TnT} \geq 14 \mathrm{ng} / \mathrm{L}$ at baseline. Therefore, only patients who had hs-TnT $\geq 14 \mathrm{ng} / \mathrm{L}$ before the first cycle died, had a stroke/TIA, or new-onset HF. Furthermore, 9 out of 13 patients with the secondary endpoint (progression of cardiac disease) had hs-TnT $\geq 14 \mathrm{ng} / \mathrm{L}$ before the first cycle $(p=0.012)$.

hs-TnT was significantly higher in patients experiencing the primary or secondary endpoints (Figure 1). AUC values were 0.909 for the prediction of the primary endpoint (with 0.796 for cardiovascular death alone), and 0.757 for the secondary endpoint (Figure 1). The best cut-offs were $14 \mathrm{ng} / \mathrm{L}$ for the primary endpoint (100\% sensitivity, $73 \%$ specificity), and again $14 \mathrm{ng} / \mathrm{L}$ for cardiovascular death (100\% sensitivity, $59 \%$ specificity) and for the secondary endpoint (sensitivity $75 \%$, specificity $77 \%$ ).

\section{Predictors of hs-TnT levels}

After assessing the correlates of increased hs-TnT (Table 1), we searched for the predictors of absolute hs-TnT levels. All variables listed in Table 1, except for $\mathrm{ICl}$ therapy, were entered in a multivariable linear regression model. The only independent predictor of ( $\log _{2}$-transformed) hs$\mathrm{TnT}$ was eGFR, but several variables approached statistical significance, namely hypertension, age, non-invasive arterial oxygen saturation, haemoglobin, posterior wall thickness, LVEF, LAVi, and tricuspid annular plane systolic excursion (Table 2).

\section{DISCUSSION}

In a cohort of patients treated with ICls, hs-TnT levels before treatment start yielded prognostic significance for the occurrence of 2 clinically relevant outcomes, namely a primary endpoint including cardiovascular death, stroke or transient ischemic attack, pulmonary embolism and new-onset HF, and a secondary endpoint consisting in the progression of cardiac involvement across stages of the CARDIOTOX classification ${ }^{38}$, both evaluated 3 months after the first cycle. 
Baseline hs-TnT $\geq 14 \mathrm{ng} / \mathrm{L}$ had $100 \%$ sensitivity and quite good specificity $(73 \%)$ for the prediction of this endpoint, and performed quite well also for the prediction of cardiovascular death alone ( $100 \%$ sensitivity, $59 \%$ specificity), as well as the secondary endpoint (sensitivity $75 \%$, specificity $77 \%)$. When searching for determinants of hs-TnT among patient characteristics before treatment start, eGFR emerged as an independent predictor of hs-TnT levels.

With the possible exception of skeletal myopathy, which is characterized by the re-expression of the cardiac isoform of troponin $T$ in the damaged and regenerating muscle ${ }^{41,42}$, elevation of hs$\mathrm{TnT}$ is a specific indicator of cardiac damage. Moreover, the upper reference limit (URL; $14 \mathrm{ng} / \mathrm{L}$ for hs-TnT) corresponds to $\mathrm{TnT}$ content in around $40 \mathrm{mg}$ of myocardial tissue ${ }^{43}$. Therefore, this highly sensitive assay is able to reveal the ongoing damage to even very limited amounts of myocardial tissue, and then disclose subclinical cardiac damage in a very early preclinical stage, likely accounting for the prognostic value of hs-TnT even in asymptomatic subjects from the general population ${ }^{21}$.

In our study, enrolling patients with not even subclinical LV dysfunction, the median hs-TnT at baseline was just $12 \mathrm{ng} / \mathrm{L}$. The risk of the primary composite endpoint increased with hs-TnT levels, with the best cut-off corresponding to the URL. The high proportion of patients experiencing manifestations of cardiac disease over just 3 months, including 2 cardiac deaths out of a total number of 3 deaths, is worth noting, and highlights the crucial need for an accurate stratification of the cardiovascular risk. Interestingly, patients with baseline hs-TnT $\geq 14 \mathrm{ng} / \mathrm{L}$ have also a greater likelihood of progressing from normal or mild cardiac involvement to mild, moderate, or even severe disease, according to the CARDIOTOX classification ${ }^{38}$. Since the limited number of events did not allow to perform multivariable analyses, and then to account for potential confounders, we searched for predictors of baseline hs-TnT, looking for close correlations that could explain the good performance of hs-TnT in risk prediction, and also for variables that could replace this biomarker as outcome predictors. Only one independent predictor of hs-TnT emerged, namely eGFR, in agreement with the role of kidneys in clearing troponin from the circulation ${ }^{44}$. Several other variables approached statistical significance, but, overall, hs-TnT seemed to convey an information on myocardial vulnerability that was additive to other variables.

Our results suggest that hs-TnT should be assessed before the start of $\mathrm{ICI}$ treatment even in patients that have been screened for HF or asymptomatic LV dysfunction (defined as LVEF $<50 \%$ ). Patients with hs-TnT $\geq 14 \mathrm{ng} / \mathrm{L}$ should be considered at high risk of developing clinically evident cardiac disease and to progress across stages of the CARDIOTOX classification over 3 months. The prognostic impact of raised hs-TnT should be verified in larger cohorts with longer follow-up periods. Importantly, hs-TnT testing should not be perceived as a strategy to select 
patients who should not receive $\mathrm{ICI}$ therapy, but as a way to identify patients with a higher risk, who may benefit from a closer follow-up (possibly integrated by serial hs-TnT measurements) to promptly detect manifestations of cardiac disease, as well as cardioprotective strategies such as therapy with statins or beta-blockers ${ }^{45}$, or a more intensive control of cardiovascular risk factor. Several limitations must be acknowledged to this preliminary, hypothesis-generating study. First, the small population size and short follow-up duration led to a limited number of events, requiring the assessment of a heterogeneous primary endpoint, and preventing the inclusion of further variables in the multivariable model. Furthermore, we performed a cumulative assessment of different therapies and tumour types, even though the risk of cardiac disease manifestations could vary according to the specific $\mathrm{ICl}$, the site of the primary tumour (lungs versus pleural spaces), or different tumour types. Second, we focused on the prognostic value of baseline values of hs-TnT, not considering changes in hs-TnT across the first cycle or during the following cycles. Third, we did not assess the prognostic value of baseline troponin I, as measurable with one of the many available hs assays currently available. Furthermore, natriuretic peptide values were not systematically measured at baseline and during follow-up, and were then not considered in the present analysis. Fourth, follow-up evaluation did not include cardiovascular magnetic resonance examinations, which could have allowed to detect subclinical forms of myocarditis.

In conclusion, in a cohort of patients treated with ICls, baseline hs-TnT predicts a composite cardiovascular endpoint and the progression of cardiac involvement over 3 months, with $14 \mathrm{ng} / \mathrm{L}$ as the best cut-off for outcome prediction.

\section{Acknowledgements: none}

No disclosures or conflict of interest related to the content of this manuscript by any of the coauthors.

\section{REFERENCES}

This article is protected by copyright. All rights reserved 
1. Pitt JM, Vétizou $M$, Daillère $R$, et al. Resistance mechanisms to immune-checkpoint blockade in cancer: tumor-intrinsic and -extrinsic factors. Immunity 2016;44:1255-1269.

2. Hurst $\mathrm{JH}$. Cancer immunotherapy innovator James Allison receives the 2015 LaskerDeBakey clinical medical research award. J Clin Invest 2015;125:3732-3736.

3. Hodi FS, O'Day SJ, McDermott DF, et al. Improved survival with ipilimumab in patients with metastatic melanoma. N Engl J Med 2010;363:711-723.

4. Tajiri K, leda M. Cardiac complications in immune checkpoint inhibition therapy. Front Cardiovasc Med 2019;6:3.

5. Postow MA, Sidlow R, Hellmann MD. Immune-related adverse events associated with immune checkpoint blockade. N Engl J Med 2018;378:158-168.

6. Li B, Chan HL, Chen P. Immune checkpoint inhibitors: basics and challenges. Curr Med Chem 2019;26:3009-3025.

7. Spallarossa $P$, Meliota $G$, Brunelli $C$, et al. Potential cardiac risk of immune-checkpoint blockade as anticancer treatment: What we know, what we do not know, and what we can do to prevent adverse effects. Med Res Rev 2018;38:1447-1468.

8. Johnson DB, Balko JM, Compton ML, et al. Fulminant myocarditis with combination immune checkpoint blockade. N Engl J Med 2016;375:1749-1755.

9. Salem J-E, Manouchehri A, Moey M, et al. Cardiovascular toxicities associated with immune checkpoint inhibitors: an observational, retrospective, pharmacovigilance study. Lancet Oncol 2018;19:1579-1589.

10. Mahmood SS, Fradley MG, Cohen JV, et al. Myocarditis in patients treated with immune checkpoint inhibitors. J Am Coll Cardiol 2018;71:1755-1764.

11. Neilan TG, Rothenberg ML, Amiri-Kordestani L, et al. Myocarditis associated with immune checkpoint inhibitors: an expert consensus on data gaps and a call to action. Oncologist 2018;23:874-878.

12. Nykl R, Fischer O, Vykoupil K, Taborsky M. A unique reason for coronary spasm causing temporary ST elevation myocardial infarction (inferior STEMI) - systemic inflammatory response syndrome after use of pembrolizumab. Arch Med Sci Atheroscler Dis 2017;2:100102.

13. Roth ME, Muluneh $B$, Jensen $B C$, et al. Left ventricular dysfunction after treatment with ipilimumab for metastatic melanoma. Am J Ther 2016;23:e1925-e1928.

14. Anderson RD, Brooks M. Apical takotsubo syndrome in a patient with metastatic breast carcinoma on novel immunotherapy. Int J Cardiol 2016;222:760-761.

15. Heinzerling L, Ott PA, Hodi FS, et al. Cardiotoxicity associated with CTLA4 and PD1 blocking immunotherapy. J ImmunoTher Cancer 2016;4:50. 
16. Ederhy S, Cautela J, Ancedy Y, Escudier M, Thuny F, Cohen A. Takotsubo-Like syndrome in cancer patients treated with immune checkpoint inhibitors. JACC Cardiovasc Imaging 2018;11:1187-1190.

17. Behling J, Kaes J, Munzel T, Grabbe S, Loquai C. New-onset third-degree atrioventricular block because of autoimmune-induced myositis under treatment with antiprogrammed cell death-1 (nivolumab) for metastatic melanoma. Melanoma Res 2017;27:155.

18. Gibson R, Delaune J, Szady A, Markham M. Suspected autoimmune myocarditis and cardiac conduction abnormalities with nivolumab therapy for non-small cell lung cancer. BMJ Case Rep 2016;2016:bcr2016216228.

19. Januzzi JL Jr, Filippatos G, Nieminen M, Gheorghiade M. Troponin elevation in patients with heart failure: on behalf of the third Universal Definition of Myocardial Infarction Global Task Force: Heart Failure Section. Eur Heart J 2012;33:2265-2271.

20. Aimo A, Januzzi JL Jr, Vergaro $G$, et al. Prognostic value of high-sensitivity troponin $t$ in chronic heart failure: an individual patient data meta-analysis. Circulation 2018;137:286297.

21. van der Linden N, Klinkenberg LJ, Bekers O, et al. Prognostic value of basal high-sensitive cardiac troponin levels on mortality in the general population: A meta-analysis. Medicine (Baltimore) 2016;95:e5703.

22. Michel L, Mincu RI, Mahabadi AA, et al. Troponins and brain natriuretic peptides for the prediction of cardiotoxicity in cancer patients: a meta-analysis. Eur $\mathrm{J}$ Heart Fail 2020;22:350-361.

23. Demissei BG, Hubbard RA, Zhang L, et al. Changes in cardiovascular biomarkers with breast cancer therapy and associations with cardiac dysfunction. J Am Heart Assoc 2020;9:e014708.

24. Sarocchi M, Grossi F, Arboscello E, et al. Serial troponin for early detection of nivolumab cardiotoxicity in advanced non-small cell lung cancer patients. Oncologist 2018;23:936-942.

25. Blaes $\mathrm{AH}$, Rehman A, Vock DM, et al. Utility of high-sensitivity cardiac troponin $\mathrm{T}$ in patients receiving anthracycline chemotherapy. Vasc Health Risk Manag 2015;11:591-594.

26. Katsurada K, Ichida M, Sakuragi M, et al. High-sensitivity troponin $T$ as a marker to predict cardiotoxicity in breast cancer patients with adjuvant trastuzumab therapy. Springerplus 2014;3:620.

27. Vaddepally RK, Kharel P, Pandey R, et al. Review of indications of FDA-approved immune checkpoint inhibitors per NCCN guidelines with the level of evidence. Cancers 2020;12:738.

This article is protected by copyright. All rights reserved 
26. Emdin M, Passino C, Prontera C, et al. Cardiac natriuretic hormones, neurohormones, thyroid hormones and cytokines in normal subjects and patients with heart failure. Clin Chem Lab Med 2004;42:627-636.

27. Levey AS, Stevens LA. Estimating GFR using the CKD Epidemiology Collaboration (CKDEPI) creatinine equation: more accurate GFR estimates, lower CKD prevalence estimates, and better risk predictions. Am J Kidney Dis 2010;55:622-627.

28. Giannitsis E, Kurz K, Hallermayer K, Jarausch J, Jaffe AS, Katus HA. Analytical validation of a high-sensitivity cardiac troponin T assay. Clin Chem 2010;56:254-261.

29. Lang RM, Badano LP, Mor-Avi V, et al. Recommendations for cardiac chamber quantification by echocardiography in adults: an update from the American Society of Echocardiography and the European Association of Cardiovascular Imaging. J Am Soc Echocardiogr 2015;28:1-39.e14.

30. Nagueh SF, Smiseth OA, Appleton CP, et al. Recommendations for the evaluation of left ventricular diastolic function by echocardiography: an update from the American Society of Echocardiography and the European Association of Cardiovascular Imaging. Eur Heart $J$ Cardiovasc Imaging 2016.

31. Rudski LG, Lai WW, Afilalo J, et al. Guidelines for the echocardiographic assessment of the right heart in adults: a report from the American Society of Echocardiography endorsed by the European Association of Echocardiography, a registered branch of the European Society of Cardiology, and the Canadian Society of Echocardiography. J Am Soc Echocardiogr 2010;23:685-713.

32. Lancellotti P, Tribouilloy C, Hagendorff A, et al. Recommendations for the echocardiographic assessment of native valvular regurgitation: an executive summary from the European Association of Cardiovascular Imaging. Eur Heart J Cardiovasc Imaging 2013;14:611-644.

33. Baumgartner $\mathrm{H}$, Hung $\mathrm{J}$, Bermejo $\mathrm{J}$, et al. Recommendations on the echocardiographic assessment of aortic valve stenosis: a focused update from the European Association of Cardiovascular Imaging and the American Society of Echocardiography. J Am Soc Echocardiogr 2017;30:372-392.

34. Badano LP, Kolias TJ, Muraru D, et al. Standardization of left atrial, right ventricular, and right atrial deformation imaging using two-dimensional speckle tracking echocardiography: a consensus document of the EACVI/ASE/Industry Task Force to standardize deformation imaging. Eur Heart J Cardiovasc Imaging 2018;19:591-600.

35. Plana JC, Galderisi M, Barac A, et al. Expert consensus for multimodality imaging evaluation of adult patients during and after cancer therapy: a report from the American 
Society of Echocardiography and the European Association of Cardiovascular Imaging. J Am Soc Echocardiogr 2014;27:911-939.

36. López-Sendón J, Álvarez-Ortega C, Zamora Auñon P, et al. Classification, prevalence, and outcomes of anticancer therapy-induced cardiotoxicity: the CARDIOTOX registry. Eur Heart J 2020;41:1720-1729.

37. Majem M, García-Martínez E, Martinez M, et al. SEOM clinical guideline for the management of immune-related adverse events in patients treated with immune checkpoint inhibitors (2019). Clin Transl Oncol 2020;22:213-222.

38. Vittinghoff E, McCulloch CE. Relaxing the rule of ten events per variable in logistic and Cox regression. Am J Epidemiol 2007;165:710-718.

39. Rittoo D, Jones A, Lecky B, Neithercut D. Elevation of cardiac troponin T, but not cardiac troponin I, in patients with neuromuscular diseases: implications for the diagnosis of myocardial infarction. J Am Coll Cardiol 2014;63:2411-2420.

40. Schmid J, Liesinger L, Birner-Gruenberger R, et al. Elevated cardiac troponin $T$ in patients with skeletal myopathies. J Am Coll Cardiol 2018;71:1540-1549.

41. Marjot J, Kaier TE, Martin ED, et al. Quantifying the release of biomarkers of myocardial necrosis from cardiac myocytes and intact myocardium. Clin Chem 2017;63:990-996.

42. Fridén V, Starnberg K, Muslimovic A, et al. Clearance of cardiac troponin $\mathrm{T}$ with and without kidney function. Clin Biochem 2017;50:468-474.

43. Kalam K, Marwick TH. Role of cardioprotective therapy for prevention of cardiotoxicity with chemotherapy: a systematic review and meta-analysis. Eur J Cancer 2013;49:2900-2909. 


\section{LEGEND TO FIGURES}

Figure 1. High-sensitivity troponin $\mathrm{T}$ (hs-TnT) for outcome prediction in cancer patients on therapy with immune checkpoint inhibitors. 
Table 1. Population characteristics.

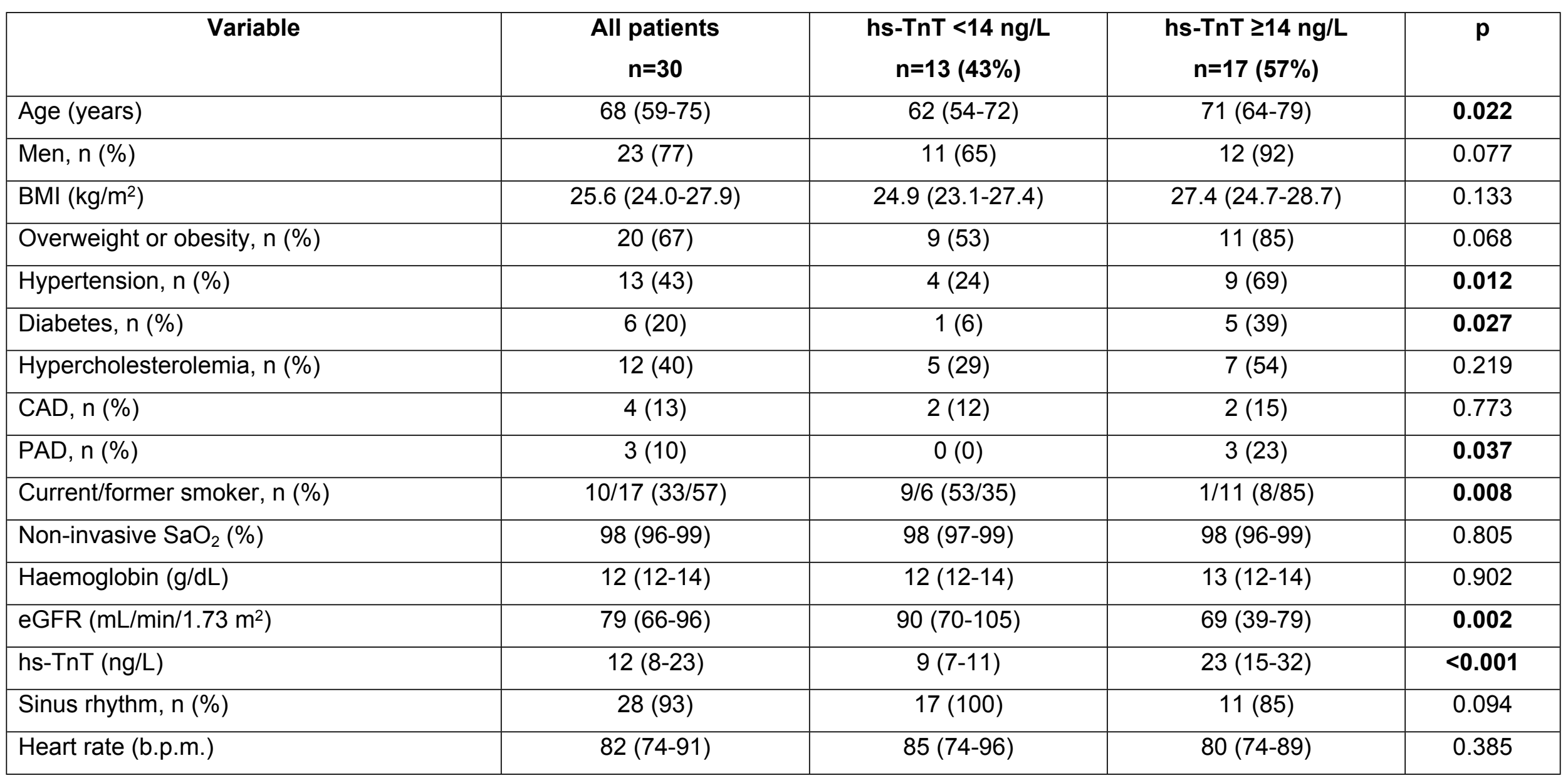




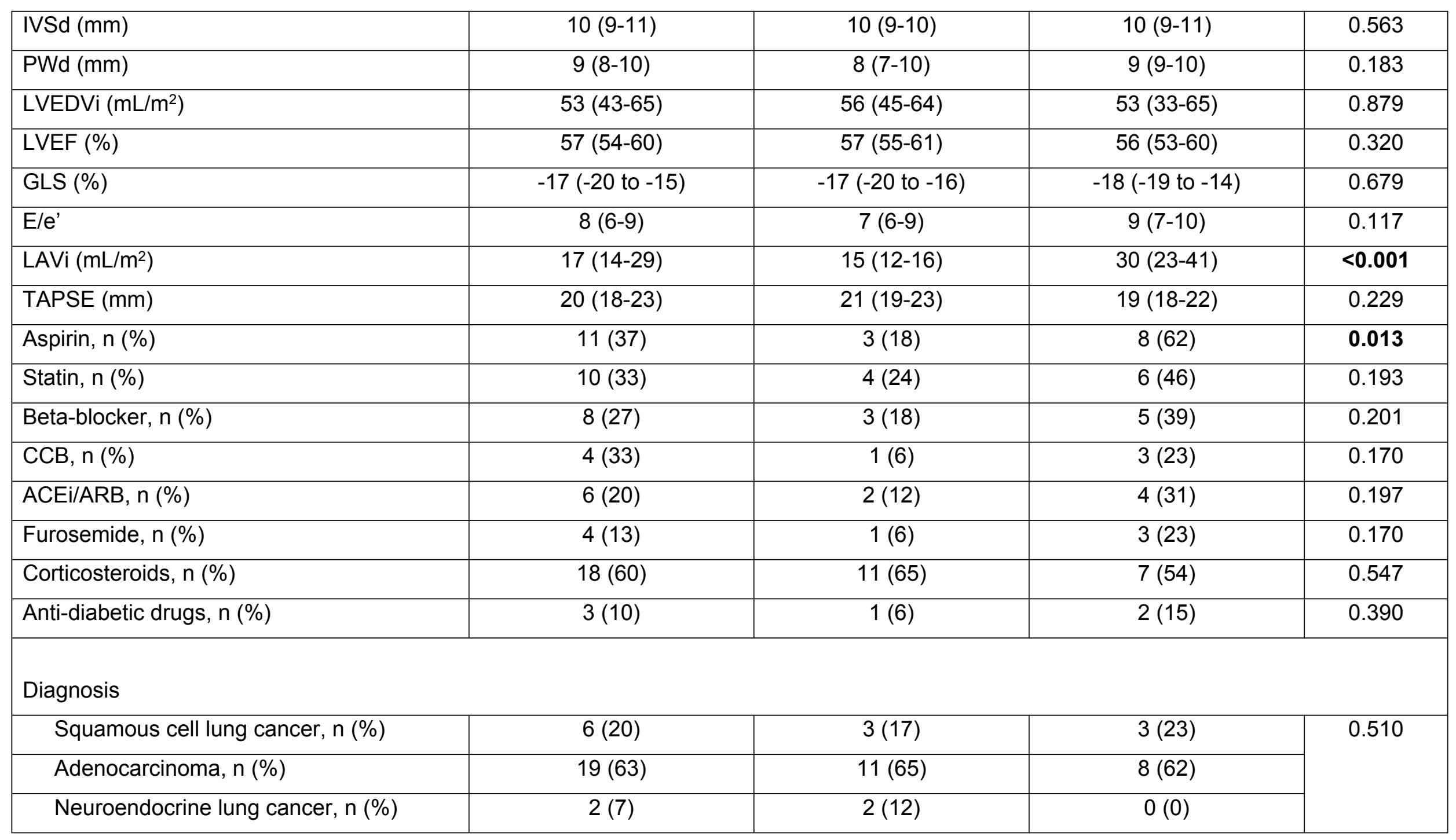




\begin{tabular}{|l|c|r|r|}
\hline \multicolumn{1}{|c|}{ Pleural mesothelioma, $\mathrm{n}(\%)$} & $3(10)$ & $1(6)$ & $2(15)$ \\
\hline Previous chemotherapy, $\mathrm{n}(\%)$ & $15(50)$ & $10(59)$ & $5(39)$ \\
\hline $\mathrm{ICI}$ & & & 0.269 \\
\hline Pembrolizumab, $\mathrm{n}(\%)$ & $15(23)$ & $4(24)$ & $7(54)$ \\
\hline Nivolumab, $\mathrm{n}(\%)$ & $8(12)$ & $3(18)$ & 0.461 \\
\hline Durvalumab, $\mathrm{n}(\%)$ & $3(5)$ & $2(12)$ & $2(15)$ \\
\hline Atezolizumab, $\mathrm{n}(\%)$ & $4(6)$ & \\
\hline
\end{tabular}

ACEi/ARB, angiotensin converting enzyme inhibitor/angiotensin receptor blocker; BMI, body mass index; CAD, coronary artery disease; CCB, calcium channel blocker; eGFR, estimated glomerular filtration rate; GLS, global longitudinal strain; hs-TnT, high-sensitivity troponin T; ICI, immune checkpoint inhibitor; IVSd, interventricular septum thickness in diastole; LAVi, left atrial volume index; LVEDVi, left ventricular end-diastolic volume index; LVEF, left ventricular ejection fraction; PAD, peripheral artery disease; PWd, posterior wall thickness in diastole; SaO ${ }_{2}$, arterial oxygen saturation; TAPSE, tricuspid annular plane systolic excursion. 
Table 2. Predictors of high-sensitivity troponin $T$ (hs-TnT) levels: linear regression analysis.

\begin{tabular}{|l|c|c|}
\hline \multicolumn{1}{|c|}{ Variable } & $\begin{array}{c}\text { Univariable } \\
\text { analysis }\end{array}$ & $\begin{array}{c}\text { Multivariable } \\
\text { analysis }\end{array}$ \\
\cline { 2 - 3 } & $\mathbf{p}$ & $\mathbf{p}$ \\
\hline Age & 0.059 & 0.844 \\
\hline Men & 0.051 & 0.182 \\
\hline BMI & 0.906 & - \\
\hline Hypertension & 0.027 & 0.531 \\
\hline Diabetes & 0.003 & 0.140 \\
\hline Hypercholesterolemia & 0.311 & - \\
\hline CAD & 0.778 & - \\
\hline PAD & 0.299 & - \\
\hline Current/former smoker & 0.172 & - \\
\hline Non-invasive SaO ${ }_{2}$ & 0.320 & - \\
\hline Haemoglobin & 0.934 & - \\
\hline eGFR & $<0.001$ & - \\
\hline Sinus rhythm & 0.867 & - \\
\hline Heart rate & 0.601 & - \\
\hline IVSd & 0.944 & - \\
\hline PWd & 0.330 & - \\
\hline LVEDVi & 0.401 & - \\
\hline LVEF & 0.661 & - \\
\hline GLS & 0.743 & - \\
\hline E/e' & & - \\
\hline LAVi & 0.001 & - \\
\hline TAPSE & 0.249 & - \\
\hline & & - \\
\hline
\end{tabular}

$\mathrm{Hs}$-TnT levels were $\log _{2}$-transformed. Univariable predictors with $\mathrm{p}<0.10$ were entered into a multivariable model, to search for independent predictors of hs-TnT. ACEi/ARB, angiotensin converting enzyme inhibitor/angiotensin receptor blocker; BMI, body mass index; CAD, coronary artery disease; eGFR, estimated glomerular filtration rate; GLS, global longitudinal strain; IVSd, 
interventricular septum thickness in diastole; LAVi, left atrial volume index; LVEDVi, left ventricular end-diastolic volume index; LVEF, left ventricular ejection fraction; PAD, peripheral artery disease; PWd, posterior wall thickness in diastole; $\mathrm{SaO}_{2}$, arterial oxygen saturation; TAPSE, tricuspid annular plane systolic excursion. 
Figure 1. High-sensitivity troponin $\mathrm{T}$ (hs-TnT) for outcome prediction in cancer patients on therapy with immune checkpoint inhibitors.
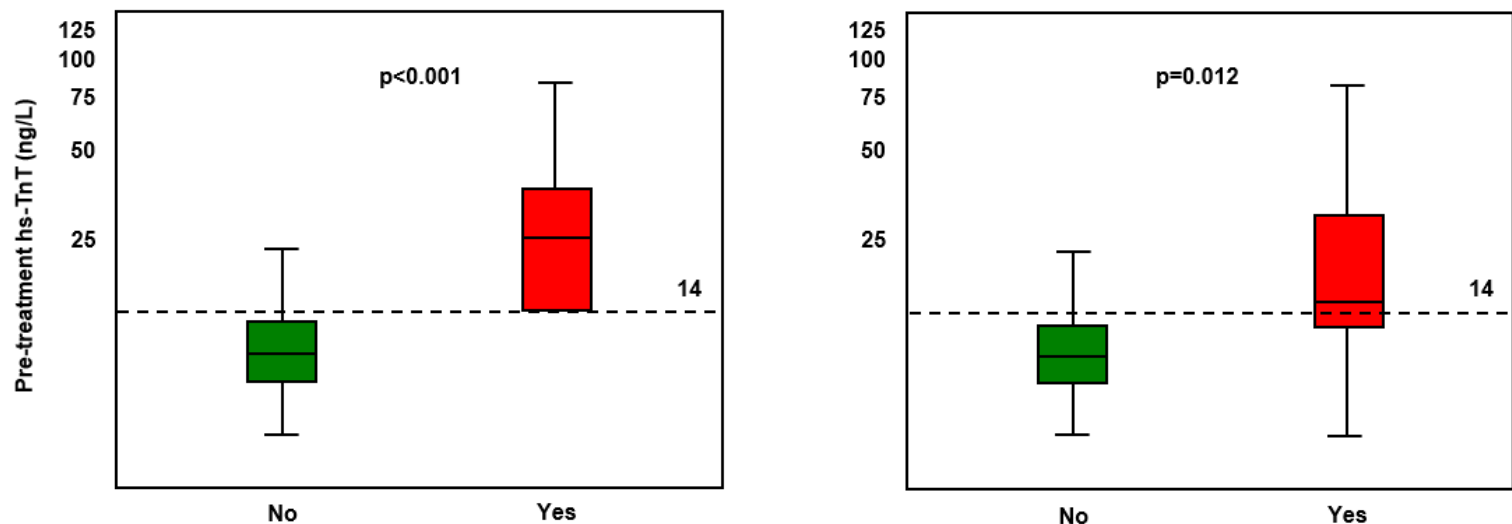

CV death, non-fatal MI, stroke/TIA, PE, HF at 3 months
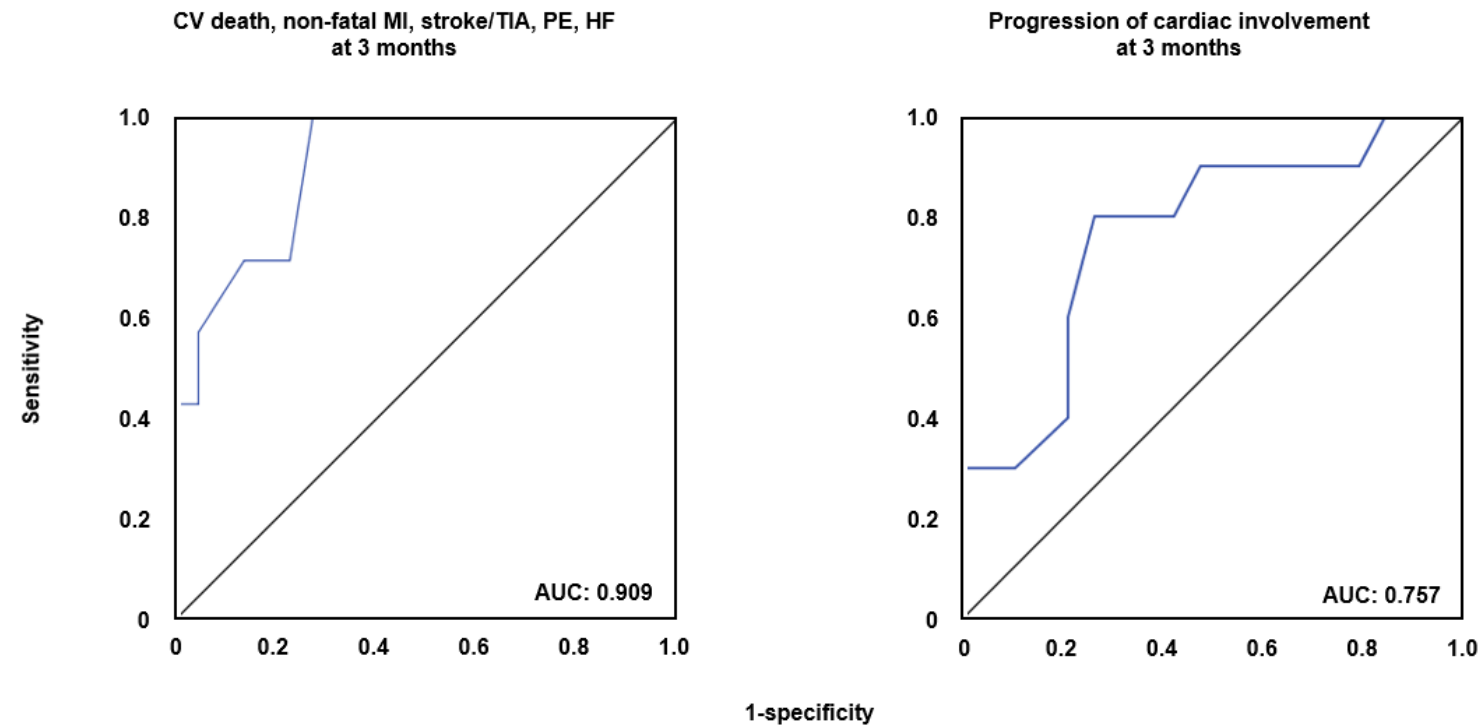

Above: comparisons between hs-TnT levels in patients experiencing or not experiencing the primary and secondary endpoint. Below: area under the curve values for hs-TnT for the prediction of the two endpoints. AUC, area under the curve; CV, cardiovascular; HF, heart failure; MI, myocardial infarction; PE, pulmonary embolism; TIA, transient ischemic attack. 